\title{
Provider management of equivocal cervical cancer screening results among underserved women, 2009-2011: follow-up of atypical squamous cells of undetermined significance
}

\author{
Meg Watson, Vicki Benard, and Lavinia Lin \\ Epidemiology and Applied Research Branch, Division of Cancer Prevention and Control, Centers \\ for Disease Control and Prevention, 4770 Buford Hwy NE MS-F76, Atlanta, GA 30341, USA \\ Tanner Rockwell \\ Information Management Services, Inc., 3901 Calverton Boulevard, Suite 200, Calverton, MD \\ 20705, USA \\ Janet Royalty \\ Epidemiology and Applied Research Branch, Division of Cancer Prevention and Control, Centers \\ for Disease Control and Prevention, 4770 Buford Hwy NE MS-F76, Atlanta, GA 30341, USA
}

\begin{abstract}
Purpose-Reflex human papillomavirus (HPV) testing is the preferred triage option for most women diagnosed with atypical squamous cells of undetermined significance (ASC-US). This study was conducted to describe follow-up results of women with ASC-US Pap test results in the National Breast and Cervical Cancer Early Detection Program (NBCCEDP), focusing on HPV test use.

Methods-We examined the follow-up of 45,049 women in the NBCCEDP with ASC-US Pap tests during 2009-2011. Data on demographic characteristics, diagnostic procedures, and clinical outcomes were analyzed.

Results-NBCCEDP providers diagnosed 45,049 women (4.5\% of all Pap tests) with an ASCUS result. Of those, 28,271 (62.8\%) were followed with an HPV test, 3,883 (8.6\%) with a repeat Pap test, 6,592 (14.6\%) with colposcopy, and 6,303 were lost to follow-up (14.0\%). Women aged 40 and older were followed more often with an HPV test. White, black, and Asian/Pacific Islander women were followed more often with an HPV test after an ASC-US Pap compared to Hispanic and American Indian/Alaska Native (AI/AN) women. Among women with a positive HPV test on follow-up, almost $90 \%$ continued with colposcopy as recommended. AI/AN women had the highest rates of HPV positivity (55.2\%) and of no follow-up (25.0\%).
\end{abstract}

\footnotetext{
(C) Springer International Publishing Switzerland (outside the USA) 2015 mwatson2@cdc.gov.

The findings and conclusions in this report are those of the authors and do not necessarily represent the official position of the Centers for Disease Control and Prevention.

Conflict of interest The authors declared no potential conflicts of interest with respect to the research, authorship, and/or publication of this article.
} 
Conclusion-This is the first analysis describing follow-up of ASC-US Pap test results in the NBCCEDP, providing a window into current management of ASC-US results. Findings raise concerns about persistent disparities among AI/AN women. During 2009-2011, nearly two-thirds of women with an ASC-US Pap test result were followed with an HPV reflex test.

\section{Keywords}

HPV testing; ASC-US; NBCCEDP; Pap test

\section{Introduction}

Each year, more than two million women in the USA are diagnosed with a mild Pap test abnormality called "atypical squamous cells of undetermined significance" (ASC-US) [1]. ASC-US is a Pap test result classification that is ambiguous, by definition [2]. ASC-US findings are generally either indicative of reactive changes (due to irritation or minor infections) or precancerous lesions indicating human papillomavirus (HPV) infection, but can rarely raise concern that more significant lesions may be present $[2,3]$. ASC-US lesions are also subjective and depend on a pathologist's interpretation [4]. HPV-positive test results subsequent to an ASC-US result indicate a possible pre-cancer and should be evaluated with colposcopy, while HPV-negative ASC-US findings help ensure that abnormalities are likely benign [1]. Thus, reflex HPV testing serves as an ancillary test used after an ASC-US result to determine whether more follow-up is needed (in the case of a positive HPV test). The 2006 American Society for Colposcopy and Cervical Pathology (ASCCP) guidelines stated that HPV testing was the preferred follow-up method after an ASC-US Pap test result and that repeat Pap testing and immediate colposcopy were also acceptable methods [5].

The Centers for Disease Control and Prevention's (CDC) National Breast and Cervical Cancer Early Detection Program (NBCCEDP) is the only national organized cancer screening program for low-income, uninsured, and underinsured women [6]. The NBCCEDP recommends their programs follow ASCCP guidelines for management of abnormal Pap tests. The NBCCEDP began reimbursing providers for HPV tests as an option for ASC-US Pap follow-up after FDA approval in 2003 [7]; however, HPV test data were not systematically reported to CDC until NBCCEDP data collection was expanded in 2009. In this paper, we examined the follow-up of women being screened in the NBCCEDP with an ASC-US Pap result during 2009-2011 to determine how providers serving this population of underserved women are following up on ASC-US results.

\section{Methods}

$\mathrm{CDC}$ has established cooperative agreements with states, American Indian/Alaska Native tribes, and territories to provide screening, referral, and follow-up services to women through the NBCCEDP, described in detail elsewhere [6, 8]. Since the NBCCEDP's inception in 1991, CDC has collected a set of standardized data items to monitor screening, diagnostic follow-up, and treatment initiation activities [8]. Women reported demographic characteristics and prior screening history at enrollment. Providers reported dates and results of Pap tests as well as any diagnostic procedures, outcomes, and the date of treatment 
initiation if applicable. This study used data reported during January 2009-December 2011 from 50 states, the District of Columbia, 12 tribes, and five territories.

\section{Study outcomes}

We examined data on women screened by the NBCCEDP and diagnosed with an ASC-US

Pap test result, as classified by the Bethesda System [1]. Data on follow-up of ASC-US Pap tests extended for 15 months from the time of screening. We selected the women's first ASC-US Pap during the study period and excluded women who had a previous ASC-US or other abnormal Pap through the NBCCEDP to remove from the study any women potentially under management for a previous abnormality.

We calculated age based on the birth date at time of ASC-US Pap diagnosis using six age classifications: $18-20,21-29,30-39,40-49,50-64$, and 65 years or older. The age groups were selected after consideration of age-related influences on screening rates, primarily guidelines recommending screening initiation at age 21 [9] and Medicare benefits for those aged 65 years and older. Women reporting Hispanic ethnicity were classified as Hispanic regardless of race. Other women were classified as white; black; Asian, native Hawaiian, or other Pacific Islander (API); American Indian/Alaska Native (AI/AN); or multiracial.

During 2009-2011, the NBCCEDP performed Pap tests on 993,238 women, of which 45,049 (4.5\%) had an ASC-US result. This study examined the follow-up of these 45,049 women with ASC-US Pap tests, including clinical outcomes for HPV reflex tests for triage, repeat Pap tests (using the Bethesda System for reporting), and biopsy-directed colposcopies to report cervical intraepithelial neoplasia (CIN) and invasive cancers. Women were categorized into three mutually exclusive groups by follow-up procedure: repeat Pap test (with no HPV test or colposcopy associated with the initial ASC-US finding), HPV test (all women with HPV test as follow-up to ASC-US, regardless of other tests), or colposcopy (with no HPV or Pap associated with the initial ASC-US Pap). Women who did not receive any follow-up procedure after the initial ASC-US Pap test through the NBCCEDP during the study period were classified as lost to follow-up. Pearson Chi-square tests were performed to test the null hypothesis of no association between follow-up groups and age groups and the null hypothesis of no association between follow-up groups and racial/ethnic groups. The percentage of women in each follow-up group was calculated separately for each age group and racial/ethnic group. To estimate confidence limits for the follow-up group percentages by age group, SUDAAN software [10] was used to fit a polytomous logit model for follow-up group with age group as the covariate, and the percentages and confidence limits were calculated using the predictive margins method [11]. The same approach was used for the follow-up group percentages by racial/ethnic group.

\section{Results}

Of the 45,049 ASC-US Pap test results examined in this analysis, 28,271 (62.8\%) were followed with an HPV test (Fig. 1), 3,883 (8.6 \%) with a repeat Pap test, 6,592 (14.6\%) with a colposcopy (i.e., direct to colposcopy without an HPV test), and 6,303 (14.0\%) were lost to follow-up. Among the 11,951 women who had a positive HPV test result after an 
ASC-US Pap test, 10,410 (87.1\%) had a colposcopy (Fig. 1). Of these women, 4,185 (40.2 $\%$ ) had normal results, 4,839 (46.5\%) were diagnosed with CIN 1, and 1,231 (11.8\%) were diagnosed with CIN 2, CIN 3, or cervical carcinoma in situ. Invasive cervical cancers were detected among $22(0.2 \%)$ women after an ASC-US Pap with a positive reflex HPV test.

The proportion of women followed with an HPV test increased with age up to 40-49 years (Table 1). Women aged <40 were followed more often with a colposcopy than older women. Women aged 18-20 years had the highest percentage of lost to follow-up (22\%; $95 \%$ CI 18.6-25.8), while those aged 21-29 years had the lowest percentage (6.1\%; $95 \%$ CI 5.6$6.7)$; the proportion of women lost to follow-up increased with age over 30 years.

By race/ethnicity, about $65 \%$ of white, black, and API women were followed with an HPV test after an ASC-US Pap. Hispanic women (21.9\%; $95 \%$ CI 21.2-22.7) and those of multiple races (18.8\%; $95 \%$ CI 16.6-21.1) had the highest percent of colposcopy. API (13.0 \%; $95 \%$ CI 11.5-14.7) and AI/AN (12.6\%; $95 \%$ CI 11.2-14.2) women had the highest proportion of repeat Pap tests as follow-up. AI/AN women had the highest proportion of being lost to follow-up than other racial/ethnic groups. Figure 2 shows the distribution of HPV positivity (among those tested for HPV, the percent that were positive) by age and race/ethnicity. Of those followed with an HPV test, women aged 18-20 and 2129 years had the highest proportions of HPV positivity (73.6 and $82.5 \%$, respectively). By race, AI/AN women had the highest proportion of HPV positivity $(55.2 \%)$.

\section{Conclusion}

Reflex HPV testing has been adopted as a cervical cancer screening strategy nationally by organizations and providers [12, 13]. During 2009-2011, nearly two-thirds of women in the NBCCEDP with an ASC-US Pap test result were managed with a reflex HPV test $(62.8 \%)$ and $87.1 \%$ of women with HPV-positive results had additional diagnostic testing, in accordance with national guidelines. During our 3-year study period, HPV testing for follow-up of an ASC-US Pap test result was the preferred triage option according to 2006 ASCCP guidelines; other methods remained acceptable [5].

These findings that providers serving the NBCCEDP women are following national guidelines for management of abnormal cytology are consistent with other studies. Benard et al. [14] found that providers were following women in the NBCCEDP with low-grade abnormalities according to national guidelines for the study period. Additionally, national provider surveys have found that providers serving women in the NBCCEDP had similar results on screening and management beliefs and practices compared to providers who do not serve women in the NBCCEDP [15].

Updated 2012 ASCCP management guidelines continued to emphasize the move toward reflex testing for follow-up of ASC-US results, followed by colposcopy for HPV-positive women; immediate colposcopy after an ASC-US finding is no longer acceptable. These changes were based on research demonstrating that reflex testing after ASC-US results identifies most CIN 3 lesions, yet refers many fewer women to colposcopy [16, 17]. Comparing the combined percentages of CIN 3 and invasive cancer among those women 
who went immediately to colposcopy after the first ASC-US Pap test to results from those women who were triaged with an HPV test and referred to a colposcopy if HPV positive, we find very similar results ( 4.3 vs. $4.5 \%$, respectively; see Fig. 1), confirming that reflex HPV testing is effective at identifying abnormalities while lowering rates of colposcopy.

Our study documented differences in treatment patterns by race/ethnicity, which have been noted previously [14]. AI/AN women had the highest rates of HPV positivity in our study and also the highest rate of no follow-up ( $25 \%)$. AI/AN women have persistent disparities in cervical cancer incidence and mortality, despite efforts resulting in some declines in cervical cancer among this population [18]. Rates of HPV positivity among AI/AN women have not previously been shown to differ substantially from other racial/ethnic groups [19]. Reasons for higher rates of no follow-up among AI/AN women are unclear, and more research is needed to determine whether these differences are related to data collection or less follow-up among these women. The high percentage (14\%) of women with no followup in the study cohort overall is concerning; however, women in the NBCCEDP often have changes in income or insurance status, affecting their eligibility, and thus some women may have received subsequent screening and diagnostic services outside of the NBCCEDP.

Women younger than age 40 years were less often followed with a reflex HPV test in this study. Nearly one out of three women under the age of 40 in our study cohort with an ASCUS Pap test result had an immediate colposcopy, raising concerns about potential overdiagnosis or overtreatment in this group. Women in this age group, especially younger than age 30, have a low risk of developing cervical cancer and may experience particular harms from treatment of abnormal lesions during the reproductive years [20-22]. This finding regarding more colposcopies as compared to repeat Pap tests for follow-up in the younger age group has been shown previously in the NBCCEDP $[14,23]$. Women younger than age 40 are five times as likely to be enrolled in the NBCCEDP for diagnostic follow-up of an abnormal screening test performed elsewhere as women aged 40 and older (6.5 and 1.3 $\%$, respectively) (data not shown). Although we limited our cohort to women not previously screened within the NBCCEDP during the study period, some younger women may have been referred in from another screening (for instance, at a health fair) with abnormal results, which could contribute to higher rates of immediate colposcopy among younger women (if abnormal results had been reported elsewhere). Therefore, the younger women may represent a group at higher risk of HPV exposure and abnormal Pap results than those in the general population. Because many state programs direct outreach to women eligible for both breast and cervical cancer screening, the majority of the women screened in the NBCCEDP are over age 40 .

Our study had some limitations. We lacked information on follow-up screenings conducted outside of the NBCCEDP or on other factors that may influence a provider's follow-up and management plan, for example previous abnormal tests outside of NBCCEDP or current pregnancy.

This is the first analysis describing follow-up results of women with ASC-US Pap test results in the NBCCEDP, focusing on HPV test use. This large collection of clinical data provides a window into the practices of thousands of providers across the USA, operating in 
a multitude of clinical settings. Although our study took place during a period of changing guidelines, a majority of women with an ASC-US Pap test result were followed with the preferred method (62.8\% HPV test) according to guidelines and received recommended management if they were found to be HPV positive ( $87.1 \%$ colposcopy performed). CDC frequently communicates information to state programs regarding changes in screening and management guidelines. The NBCCEDP has workgroups that meet regularly to promote education and training around evidence-based guidelines. This study demonstrates that providers in NBCCEDP largely appear to be practicing according to current guidelines for follow-up of ASC-US Pap tests.

\section{Acknowledgments}

We would like to thank William Helsel and William Kammerer from Information Management Services, Inc. for their assistance with data analysis. This research was supported in part by an appointment (LL) to the Research Participation Program at the Centers for Disease Control and Prevention administered by the Oak Ridge Institute for Science and Education through an interagency agreement between the US Department of Energy and CDC.

\section{References}

1. Solomon D, Schiffman M, Tarone R, group AS. Comparison of three management strategies for patients with atypical squamous cells of undetermined significance: baseline results from a randomized trial. J Natl Cancer Inst. 2001; 93(4):293-299. [PubMed: 11181776]

2. American Cancer Society. [Accessed 12 Mar 2014] Finding cervical pre-cancers. 2014. http:// www.cancer.org/cancer/cervicalcancer/moreinformation/ cervicalcancerpreventionandearlydetection/cervical-cancer-pre vention-and-early-detection-findpre-cancer-changes

3. Gatscha RM, Abadi M, Babore S, Chhieng D, Miller MJ, Saigo PE. Smears diagnosed as ASCUS: interobserver variation and follow-up. Diagn Cytopathol. 2001; 25(2):138-140. [PubMed: 11477722]

4. Raab SS, Bishop NS, Zaleski MS. Long-term outcome and relative risk in women with atypical squamous cells of undetermined significance. Am J Clin Pathol. 1999; 112(1):57-62. [PubMed: 10396286]

5. Wright TC Jr, Massad LS, Dunton CJ, Spitzer M, Wilkinson EJ, Solomon D, American Society for C; Cervical Pathology-sponsored Consensus C. 2006 consensus guidelines for the management of women with abnormal cervical cancer screening tests. Am J Obstet Gynecol. 2007; 197(4):346355. doi:10.1016/j.ajog.2007.07.047. [PubMed: 17904957]

6. Mullen, J.; Lantz, P. Cancer Causes Control. 2014. Overview of the National Breast and Cervical Cancer Early Detection Program. doi:10.1007/s10552-015-0565-9

7. Gutman, SI. Premarket Approval of Digene Hybrid Capture 2 High-Risk HPV DNA Test. 2003. http://www.accessdata.fda.gov/cdrh_docs/pdf/p890064s009a.pdf

8. Ryerson AB, Benard VB, Major AC. Summarizing the first 12 years of partnerships and progress against breast and cervical cancer 1991-2002 national report. Mar.2014 242014.

9. Saslow D, Solomon D, Lawson HW, Killackey M, Kulasingam SL, Cain J, Garcia FA, Moriarty AT, Waxman AG, Wilbur DC, Wentzensen N, Downs LS Jr, Spitzer M, Moscicki AB, Franco EL, Stoler MH, Schiffman M, Castle PE, Myers ER, Committee A-A-ACCG. American Cancer Society, American Society for Colposcopy and Cervical Pathology, and American Society for Clinical Pathology screening guidelines for the prevention and early detection of cervical cancer. CA Cancer J Clin. 2012; 62(3):147-172. doi:10.3322/caac.21139. [PubMed: 22422631]

10. Research Triangle Institute. SUDAAN language manual, volumes 1 and 2, release 11. Research Triangle Institute, Research Triangle Park; 2012.

11. Graubard BI, Korn EL. Predictive margins with survey data. Biometrics. 1999; 55(2):652-659. [PubMed: 11318229] 
12. Roland KB, Benard VB, Greek A, Hawkins NA, Manninen D, Saraiya M. Primary care provider practices and beliefs related to cervical cancer screening with the HPV test in federally qualified health centers. Prev Med. 2013; 57(5):419-425. doi:10.1016/j.ypmed.2013.04.012. [PubMed: 23628517]

13. Apgar BS, Kittendorf AL, Bettcher CM, Wong J, Kaufman AJ. Update on ASCCP consensus guidelines for abnormal cervical screening tests and cervical histology. Am Fam Physician. 2009; 80(2):147-155. [PubMed: 19621855]

14. Benard VB, Howe W, Saraiya M, Helsel W, Lawson HW. Assessment of follow-up for low-grade cytological abnormalities in the National Breast and Cervical Cancer Early Detection Program, 2000-2005. J Low Genit Tract Dis. 2008; 12(4):300-306. doi:10.1097/LGT.0b013e31817e308e. [PubMed: 18820545]

15. Benard VB, Saraiya MS, Soman A, Roland KB, Yabroff KR, Miller J. Cancer screening practices among physicians in the national breast and cervical cancer early detection program. $J$ Womens Health (Larchmt). 2011; 20(10):1479-1484. doi:10.1089/jwh.2010.2530. [PubMed: 21774673]

16. Massad LS, Einstein MH, Huh WK, Katki HA, Kinney WK, Schiffman M, Solomon D, Wentzensen N, Lawson HW, Conference ACG. 2012 updated consensus guidelines for the management of abnormal cervical cancer screening tests and cancer precursors. J Low Genit Tract Dis. 2013; 17(5 Suppl 1):S1-S27. doi:10.1097/LGT.0b013e318287d329. [PubMed: 23519301]

17. Group A-LTS. A randomized trial on the management of low-grade squamous intraepithelial lesion cytology interpretations. Am J Obstet Gynecol. 2003; 188(6):1393-1400. [PubMed: 12824968]

18. Watson M, Benard VB, Thomas C, Brayboy A, Paisano R, Becker T. Cervical cancer incidence and mortality among American Indian and Alaska Native Women, 1999-2009. Am J Public Health. 2014; 104:S415. [PubMed: 24754650]

19. Alfonsi GA, Datta SD, Mickiewicz T, Koutsky LA, Ghanem K, Hagensee M, Kerndt P, Hsu K, Weinstock H, Shlay JC. Prevalence of high-risk HPV types and abnormal cervical cytology in American Indian/Alaska Native women, 2003-2005. Public Health Rep. 2011; 126(3):330-337. [PubMed: 21553660]

20. Benard VB, Watson M, Castle PE, Saraiya M. Cervical carcinoma rates among young females in the United States. Obstet Gynecol. 2012; 120(5):1117-1123. doi:10.1097/AOG. 0b013e31826e4609. [PubMed: 23090530]

21. Group T, Sharp L, Cotton S, Cochran C, Gray N, Little J, Neal K, Cruickshank M. After-effects reported by women following colposcopy, cervical biopsies and LLETZ: results from the TOMBOLA trial. BJOG. 2009; 116(11):1506-1514. doi:10.1111/j.1471-0528.2009.02263.x. [PubMed: 19583712]

22. Reilly R, Paranjothy S, Beer H, Brooks CJ, Fielder HM, Lyons RA. Birth outcomes following treatment for precancerous changes to the cervix: a population-based record linkage study. BJOG. 2012; 119(2):236-244. doi:10.1111/j.1471-0528.2011.03052.x. [PubMed: 21790951]

23. Trivers KF, Benard VB, Eheman CR, Royalty JE, Ekwueme DU, Lawson HW. Repeat pap testing and colposcopic biopsies in the underserved. Obstet Gynecol. 2009; 114(5):1049-1056. doi: 10.1097/AOG.0b013e3181b8fc88. [PubMed: 20168106] 


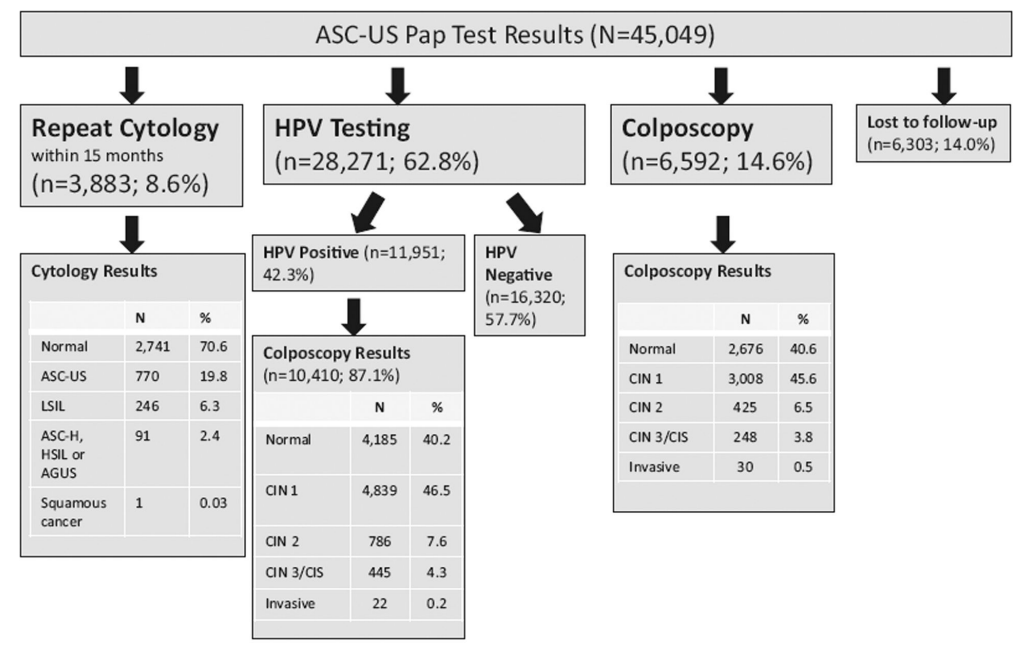

Fig. 1.

Management of women with atypical squamous cells of undetermined significance (ASCUS) on cytology $(n=45,049)$. Note of women who had a repeat cytology within 15 months, $26(0.7 \%)$ had unsatisfactory results and eight $(0.2 \%)$ had "other" results. Of HPV+ women who had a colposcopy, $133(1.3 \%)$ had "other" results. Of women who received a colposcopy without an HPV test, 205 (3.1\%) had "other" results 

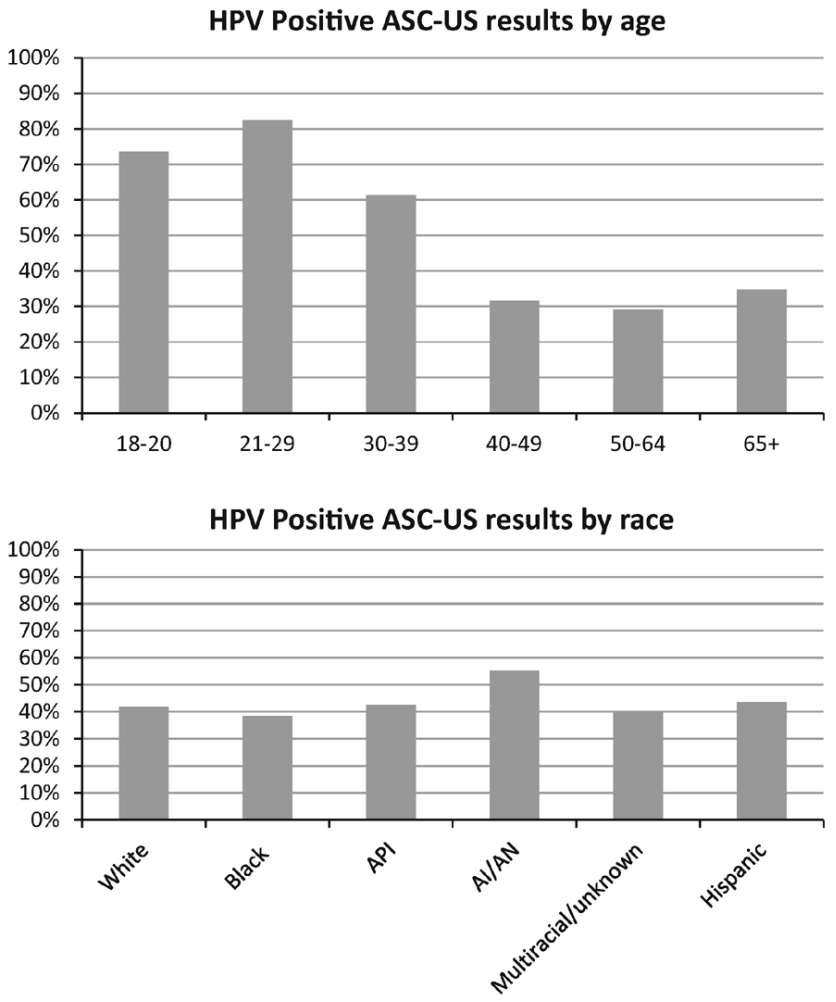

Fig. 2.

Percent of HPV-positive test results after follow-up of ASC-US Pap test, NBCCEDP, 20092011 (HPV positivity among those followed up with an HPV test) 
Table 1

Demographics and management of women with an ASC-US Pap test in the NBCCEDP, 2009-2011

\begin{tabular}{|c|c|c|c|c|c|c|c|c|c|}
\hline & \multicolumn{2}{|c|}{ Follow-up with HPV test } & \multicolumn{2}{|c|}{$\underline{\text { Immediate colposcopy }}$} & \multicolumn{2}{|c|}{ Repeat Pap } & \multicolumn{2}{|c|}{$\begin{array}{l}\text { No follow-up; lost to } \\
\text { follow-up }\end{array}$} & \multirow[t]{2}{*}{$\underline{p \text { value }}{ }^{*}$} \\
\hline & $n$ & $\%(95 \% \mathrm{CI})$ & $n$ & $\%(95 \% \mathrm{CI})$ & $n$ & $\%(95 \% \mathrm{CI})$ & $n$ & $\%(95 \% \mathrm{CI})$ & \\
\hline \multicolumn{10}{|l|}{ Age in years } \\
\hline $18-20$ & 242 & $47.5(43.2-51.9)$ & 121 & $23.8(20.3-27.7)$ & 34 & $6.7(4.8-9.2)$ & 112 & $22.0(18.6-25.8)$ & $<0.001$ \\
\hline $21-29$ & 4,310 & $53.5(52.4-54.6)$ & 2,956 & $36.7(35.7-37.8)$ & 294 & $3.7(3.3-4.1)$ & 494 & $6.1(5.6-6.7)$ & \\
\hline $30-39$ & 3,258 & $57.8(56.5-59.1)$ & 1,422 & $25.2(24.1-26.4)$ & 350 & $6.2(5.6-6.9)$ & 605 & $10.7(10.0-11.6)$ & \\
\hline $40-49$ & 10,790 & $67.6(66.8-68.3)$ & 1,211 & $7.6(7.2-8.0)$ & 1,473 & $9.2(8.8-9.7)$ & 2,496 & $15.6(15.1-16.2)$ & \\
\hline $50-64$ & 9,533 & $65.0(64.2-65.8)$ & 864 & $5.9(5.5-6.3)$ & 1,712 & $11.7(11.2-12.2)$ & 2,557 & $17.4(16.8-18.1)$ & \\
\hline $65+$ & 138 & $64.2(57.6-70.3)$ & 18 & $8.4(5.3-12.9)$ & 20 & $9.3(6.1-14.0)$ & 39 & $18.1(13.5-23.9)$ & \\
\hline \multicolumn{10}{|l|}{ Race/ethnicity } \\
\hline White & 13,998 & $65.6(65.0-66.3)$ & 2,625 & $12.3(11.9-12.8)$ & 1,914 & $9.0(8.6-9.4)$ & 2,795 & $13.1(12.7-13.6)$ & $<0.001$ \\
\hline Black & 3,925 & $64.6(63.4-65.8)$ & 699 & $11.5(10.7-12.3)$ & 480 & $7.9(7.2-8.6)$ & 971 & $16.0(15.1-16.9)$ & \\
\hline API & 1,101 & $65.3(63.0-67.5)$ & 105 & $6.2(5.2-7.5)$ & 219 & $13.0(11.5-14.7)$ & 261 & $15.5(13.8-17.3)$ & \\
\hline $\mathrm{AI} / \mathrm{AN}$ & 1,065 & $56.4(54.2-58.7)$ & 113 & $6.0(5.0-7.2)$ & 238 & $12.6(11.2-14.2)$ & 471 & $25.0(23.1-27.0)$ & \\
\hline Multiracial/unknown & 706 & $61.1(58.3-63.9)$ & 217 & $18.8(16.6-21.1)$ & 69 & $6.0(4.7-7.5)$ & 163 & $14.1(12.2-16.2)$ & \\
\hline Hispanic & 7,476 & $57.9(57.0-58.7)$ & 2,833 & $21.9(21.2-22.7)$ & 963 & $7.5(7.0-7.9)$ & 1,642 & $12.7(12.2-13.3)$ & \\
\hline
\end{tabular}

